\title{
Lessons Learned from a Radio Spectrum Coexistence Competition: A Road Map to Engagement in Informal Education of Wireless Communication
}

\author{
Mr. Joshua Alexéi García Sheridan, Virginia Tech
}

Joshua García Sheridan is a PhD student in the Department of Engineering Education at Virginia Tech. He received his Bachelor's of Science in Electrical Engineering at the University of Illinois at UrbanaChampaign. His current research work include gaming and game-like interventions in engineering education and designing interactive educational tutorials for radio engineering, with research interests in explicitly mapping childhood stages of cognitive development to engineering knowledge and skills for K-12 curricula.

\section{Dr. Richard M. Goff, Virginia Tech}

Richard M. Goff is a former aircraft structural test engineer for the Navy, a Peace Corps Volunteer, and computer entrepreneur. He holds a Ph.D. in Aerospace Engineering, and is currently an Associate Professor in the Department of Engineering Education at Virginia Tech. Richard has been teaching and engaging in research in multidisciplinary engineering design education for over twenty years. Dr. Goff is the recipient of several university teaching awards, outreach awards, and best paper awards. His passion is creating engaging learning environments by bringing useful research results and industry practices into the classroom as well as using research results to inform engineering practice.

\section{Dr. Seungmo Kim, Georgia Southern University}

Dr. Vuk Marojevic, Virginia Tech

Vuk Marojevic received his M.S. from the University of Hannover, Germany, and his Ph.D. from the Universidad Politècnica de Catalunya-Barcelona Tech, Spain, both in electrical engineering. He joined Wireless@Virginia Tech in 2013, where he is currently a Research Assistant Professor. His research interests are in software-defined radio, spectrum sharing, 4G/5G cellular technology, wireless testbeds and testing, resource management, wireless security, and engineering education with application to missioncritical networks, vehicular networks, virtualized wireless networks, green communications, the Internet of Things, and unmanned aircraft systems. Dr. Marojevic has been instructor of undergraduate and graduate level classes at the Barcelona Tech and Virginia Tech. He is a member of the IEEE, ACM, and ASEE.

\section{Dr. Carl B. Dietrich, Virginia Tech}

A licensed Professional Engineer in Virginia, Carl Dietrich earned a BS EE degree from Texas A\&M University, and MS EE and PhD EE degrees from Virginia Tech. He has taught courses in software defined radio, communications systems, electronics, and electromagnetic fields. He has also taught short courses on software defined radio since 2007, covering fundamental concepts and enabling technologies in addition to the use of open source software to develop and run SDR applications. In addition, Dr. Dietrich has performed and directed research in the areas of cognitive radio, software defined radio (SDR), multi-antenna systems, and radio wave propagation, and has authored or co-authored more than 50 peerreviewed journal and conference papers. He has worked at Virginia Tech, Bell Northern Research, and the Defense Information Systems Agency. He has served as chair of the Wireless Innovation Forum's Educational Special Interest Group, is a member of ASEE and Eta Kappa Nu, Senior Member of IEEE, and an Extra class amateur radio operator. 


\section{Lessons Learned from a Radio Spectrum Coexistence Competition: A Road Map to Engagement in Informal Education of Wireless Communication}

\section{Introduction}

The U.S. Government is in the process of implementing 2012 recommendations by the President's Council of Advisors on Science and Technology (PCAST) [1] to share federal spectrum with non-federal users, a process that is projected to result in a Trillion dollars in societal benefits as well as related employment for millions. Related competitions such as DARPA's Spectrum Challenge and Spectrum Collaboration Challenge [2] encourage innovative approaches and help prepare the STEM professionals who will develop effective spectrum sharing radios and networks as well as spectrum access systems needed to realize the PCAST vision.

We describe experience gained and lessons learned through organization of two international radio spectrum coexistence competitions. In addition, we present an assessment of these competitions based on analysis of participant survey item responses addressing self-efficacy and engagement as well as participant recommendations.

We identify and discuss several important considerations in organizing and hosting this type of competition. These considerations include infrastructure comprising both hardware and software, recruitment of sponsors, timing of funding and publicity for the competition, recruitment of participants and advisors, software and hardware documentation and ease of use, technical support, logistics for the final competition, and documentation of the competition, in particular the final competition, for use in publicizing the next year's competition, if held yearly.

\section{Facilities}

\subsection{Testbed Hardware}

One of the United States' largest cognitive radio network testbeds is located at Virginia Tech and maintained by the researchers. The testbed consists of 48 Ettus's Universal Software Radio Peripherals (USRPs), 12 nodes on each floor (Figure 1). Each USPR2 is connected through a Gigabit Ethernet directly to powerful rack server in our dedicated cluster. A total of 28 two-node racks make up the cluster, and each node contains two Xenon CPU, for a total of $8 \mathrm{CPU}$ cores and 12 GB of RAM per node. It allows running the sophisticated signal processing algorithms and SDR waveforms required to perform Cognitive Radio (CR) experiments in real time. In addition to the 24 racks connected to USRP2's, the testbed employs four racks dedicated to network management and administration. An image server provides automated re-imaging capabilities, a firewall, and a LDAP server provides security/authentication. A dedicated NFS server is employed at the user plane in order to provide researchers a private directory to store scripts, programs, and test results. Many of the experiments and demos that have been produced thus far have exploited the remote capabilities of this COgnitive Radio NETwork (CORNET) [3] 
testbed, by employing custom web interfaces, and many of the administrative tasks can now be performed using only a browser.

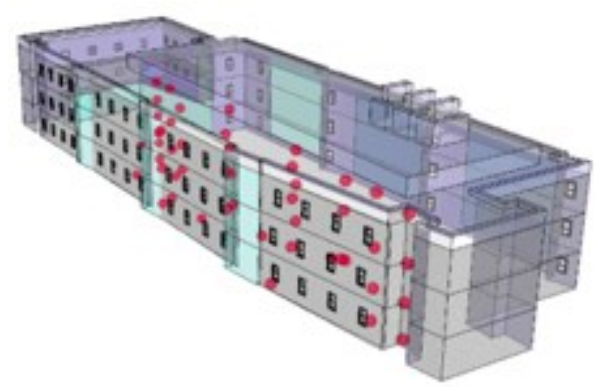

Figure 1: Locations of the 48 indoor radio nodes (USRP2) in an on-campus research building.

\subsection{Testbed Software}

The experiment management framework known as CRTS (Cognitive Radio Test System) [4] provides a flexible framework for over the air test and evaluation of cognitive radio (CR) networks. Users can rapidly define new testing scenarios involving a large number of CR's and interferers while customizing the behavior of each node individually. Execution of these scenarios is simple and the results can be quickly visualized using Octave/Matlab logs that are kept throughout the experiment.

CRTS evaluates the performance of CR networks by generating network layer traffic at each CR node and logging metrics based on the received packets. Each CR node will create a virtual network interface so that CRTS can treat it as a standard network device. Part of the motivation for this is to enable evaluation of UDP and TCP network connections. The CR object/process can be anything with such an interface. We are currently working on examples of this in standard SDR frameworks, such as GNU Radio.

A particular CR has been developed with the goal of providing a flexible generic structure to enable rapid development and evaluation of cognitive engine (CE) algorithms. This CR is being called the Extensible Cognitive Radio (ECR). In this structure, a CE is fed data and metrics relating to the current operating point of the radio. It can then make decisions and exert control over the radio to improve its performance.

The ECR uses the orthogonal frequency division multiplexing (OFDM) frame generator of liquid-dsp and uses a USRP. CRTS is available on our testbed.

Whereas CRTS and liquid-dsp allow building and controlling radios and getting raw performance indicators, another software suite, CORNET3D [5], has been developed by the group to visualize RF spectrum and radio performance. Figure 2 illustrates the building blocks of this software and a 3D visualization of RF energy over frequency and time. 

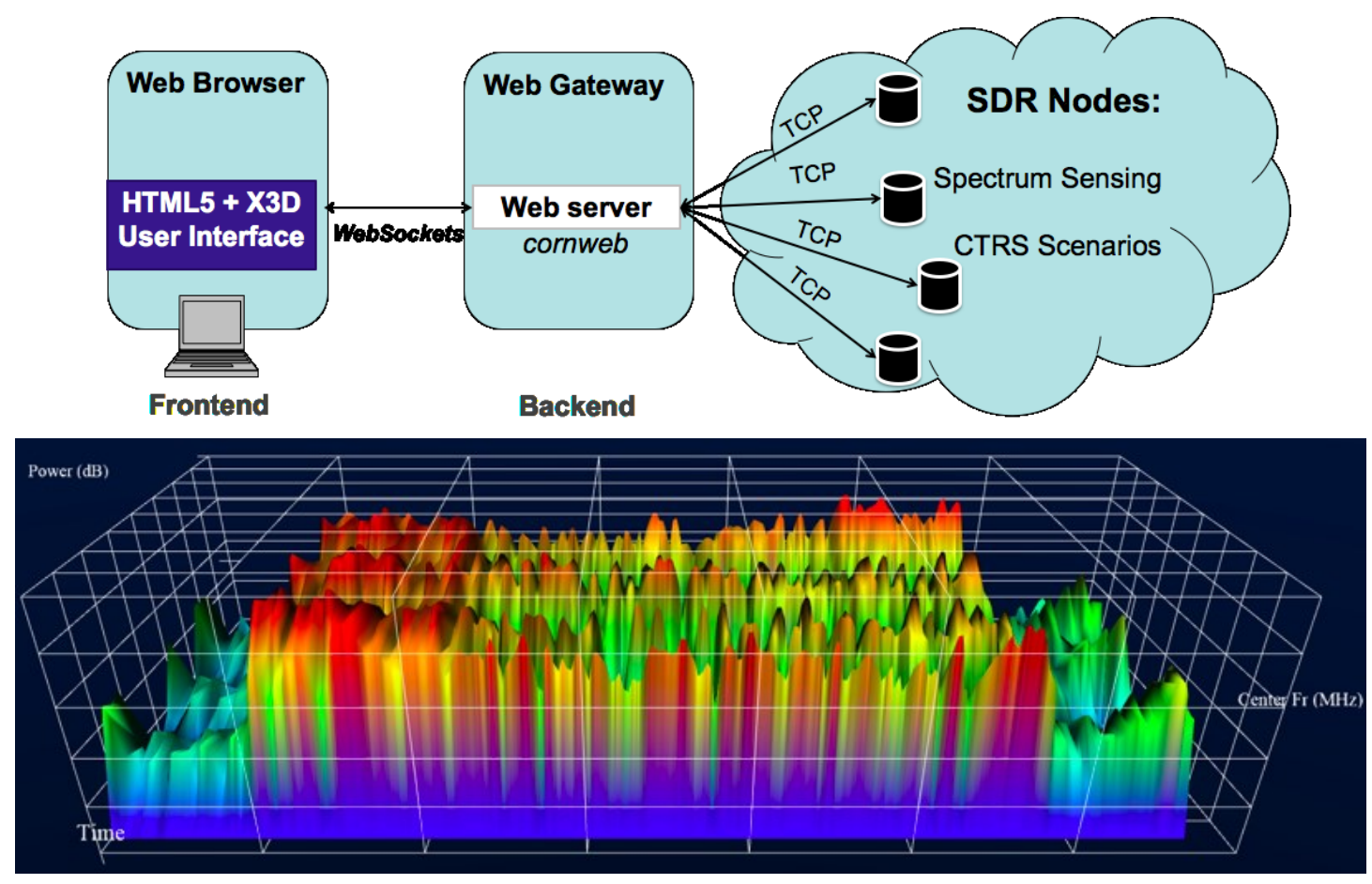

Figure 2: Data flow for CORNET3D visualization software and spectrum visualization screenshot.

\section{Description of the Competitions}

\subsection{Goals}

The competition, formally known as the Spectrum-Sharing Radio Challenge (Spectrum-ShaRC), built on our institution's experience and existing software-defined radio (SDR) experimentation infrastructure including CORNET, CRTS, and CORNET3D. CRTS enables generation of challenging radio frequency signal environments, and measures radio link performance achieved by cognitive and / or dynamic spectrum access radios as they operate in these environments. Additionally, the framework enables performance measurement of other radios that share the spectrum with the radios under test, to monitor the effects of the radios under test on other coexisting radios. The visualization tool allows users to view signals within user-selected segments of the radio spectrum. The current version of the visualization tool can also display radio link throughput and packet error rate (PER) information.

In hosting each of the two competitions, we made our testbed and software, including CRTS and source code for a configurable digital SDR waveform and autonomous controller, available to the participating teams, provided rules and clarification of the rules as needed, and provided technical support for the teams. Each competition included one or more remote preliminary rounds that were conducted remotely using the Internet-accessible testbed, and a tournamentstyle final round at our institution in which the top teams were invited to participate. 
Technical aspects of the contest were administered by graduate and undergraduate students, with staff support for publicity and development of a web site as well as administration of registration, travel arrangements, and reimbursement.

The intended final products were enhanced hands-on experience and new knowledge of the undergraduate and graduate student participants in the areas of wireless communications, software-defined radio, cognitive radio, and spectrum sharing / spectrum access, as well as a foundation for an ongoing contest that will use continuously evolving tools to enable educating generations of undergraduate and graduate electrical and computer engineering students in wireless communications using relevant scenarios.

\subsection{Activities}

Activities related to the competition included development of an open-source, ready-to-run reference waveform implemented using an open source SDR software toolkit, with associated code for an autonomous controller. Contest participants' task was to modify the controller for the reference waveform to optimize the ability of their teams' radios to transmit data in a challenging radio frequency signal environment representative of a shared radio spectrum band. Additionally, the organizers developed operational scenarios to test the participants' radios, developed a method of scoring participants' solutions, updated the visualization tool with the goal of achieving a "spectator-friendly" experience for the final round, and gave participants time-shared access to CORNET and CRTS so the teams could design and run their own tests on their radios and controllers.

Examples of operational scenarios used to test participants' radios in the preliminary rounds included:

1. Operation in a noise-limited channel with no interfering radio signals

2. Operation of the participants' radios as secondary users (SU) that must maximize performance of their own link (measured by throughput).

3. A variation of the above scenario in which participants' radios are evaluated based on both their own link performance and lack of negative impact on throughput of primary user $(\mathrm{PU})$ radio links that share the same spectrum band.

In the final-round tournament, the participants' radios operated concurrently in a head-to-head competition, within radio environments that also included other transmitters.

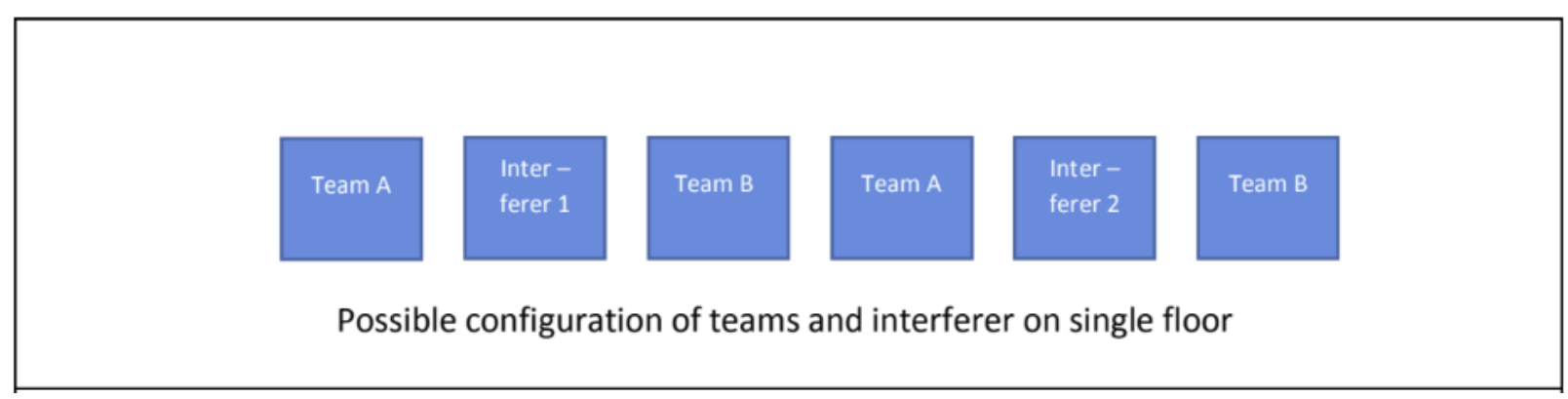

Figure 3. Interleaving of teams' radios to create a challenging signal environment. 
The following illustrations depict possible interferer behaviors and experiment setups:
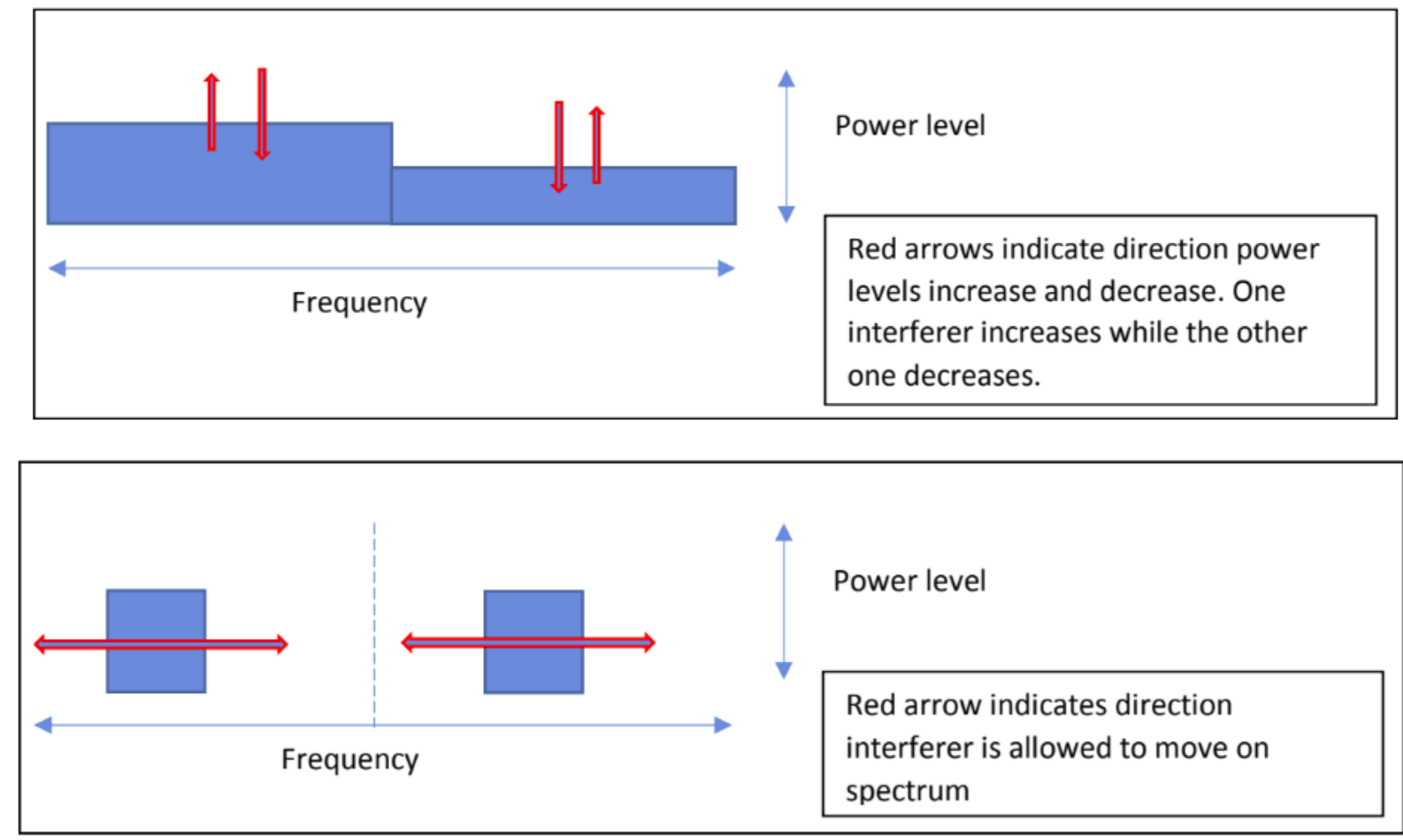

Figure 4. Possible behaviors of coexisting / interfering signals during experiments.

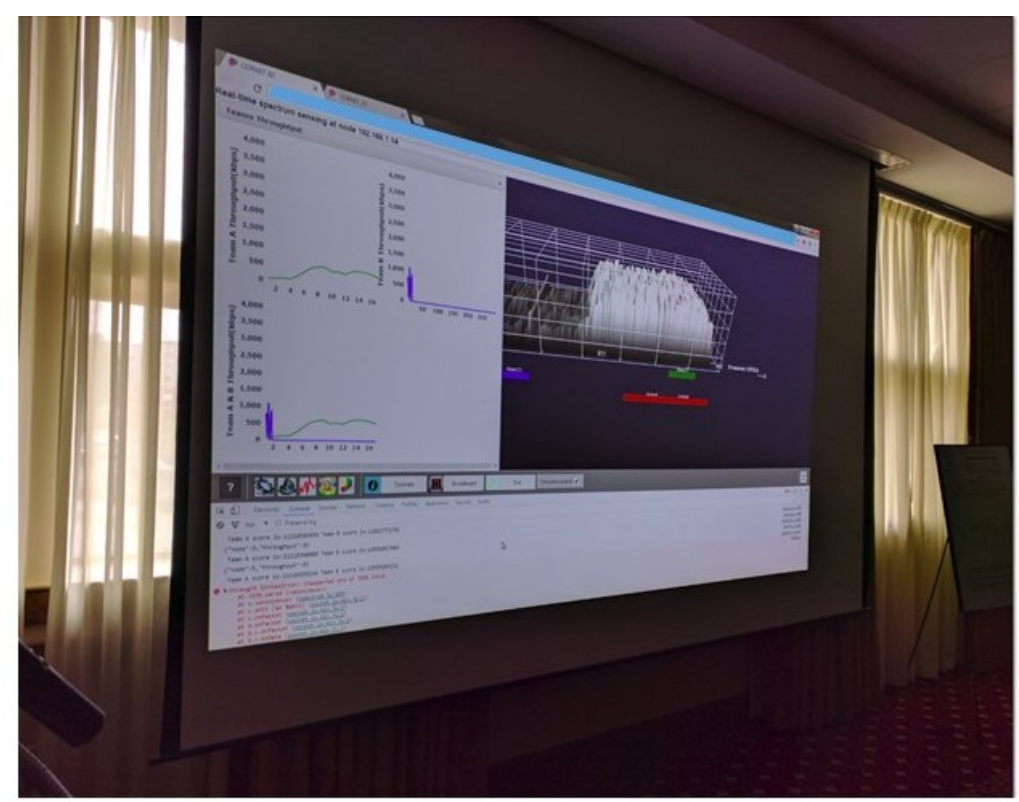

Figure 5. From the final competition in 2017: Large projection screen showing real time performance of participants' radios. Participants and spectators observe instantaneous throughput and cumulative throughput as well as a three-dimensional view of spectrum showing the interferer. 


\subsection{Participation in the Competition}

Seventeen teams consisting of 88 total participants entered the competition during the 2015-16 academic year. Of these, six teams were invited and attended the final competition. The 2016-17 competition began with six teams registering and of these, two teams completed all preliminary rounds and were invited to the final round. The 2016-17 academic year coincided with the start of the DARPA Spectrum Collaboration Challenge (SC2). SC2 included large prizes, wide publicity, and financial support for some teams, and thus may have attracted some teams who would have otherwise registered for our competition.

\section{Analysis}

\subsection{Tools}

To assess the educational value of the competition, a participant survey was crafted based on several validated Likert scale instruments used for constructs of motivation [7], self-efficacy [8], and engagement [9]. This assessment was carried out under the protocol approved by the Institutional Review Board at Virginia Tech (IRB 15-464 Cognitive Radio Contest Assessment). To ensure that the survey would not present a demotivating prospect of long fill-in times, the pool of items for the survey is composed of a limited number of the total items from these vetted instruments representing the constructs. Having been modified in diction to refer specifically to radio engineering, cognitive radio, and spectrum sharing (e.g. "Answer on the scales provided how much you agree or disagree with the following responses to this question: Why did you participate in this competition?" followed by Item 1: "Because I think that participating in this competition will help me better prepare for the career I have chosen."), the items highlight actionable affective outcomes of this activity. Using these measures, future programming and outreach for subsequent iterations of the competition could be informed by how students felt about what they were doing.

The survey uses 7-point Likert scaling ( 0 for strong disagreement, 6 for strong agreement) for a total of 30 items related to the aforementioned constructs. In addition, the survey includes free answer items for participants to respond with feedback about various aspects of the competition. The survey was delivered through an online Qualtrics form using anonymous links. Qualtrics is a web-based survey development and delivery system that stores responses in data formats such as .sav, which makes the data compatible with SPSS software.

\section{$\underline{\text { Data Processing/Cleaning }}$}

SPSS was used to perform data cleaning and statistical analysis for the Likert-based portion of the participant survey. Individual items that indicated the same constructs as defined in their instruments of origin were averaged together for each participant.

Codes for said constructs as presented in data tables to follow are presented and defined below: 
Table 1. Survey Codes and Definitions

\begin{tabular}{|c|c|}
\hline Code & Definition \\
\hline IM_KNOW & $\begin{array}{l}\text { Internal Motivation - To know, the student innately derives satisfaction } \\
\text { from learning new things }\end{array}$ \\
\hline IM_ACCOMP & $\begin{array}{l}\text { Intrinsic Motivation - Toward accomplishment, the student is driven } \\
\text { abstractly towards academic excellence as a result of their learning }\end{array}$ \\
\hline IM_STIMUL & $\begin{array}{l}\text { Intrinsic Motivation - Seeking stimulation, the student seeks the intense } \\
\text { sensations they feel while engaged in learning }\end{array}$ \\
\hline EM_EXTREG & $\begin{array}{l}\text { Extrinsic Motivation - External Regulation, other factors outside of the } \\
\text { student are forcing them to learn and perform }\end{array}$ \\
\hline EM_INTRO & $\begin{array}{l}\text { Extrinsic Motivation - Introjected, the student is abstractly asserting } \\
\text { themselves to the external world through learning and performance }\end{array}$ \\
\hline EM_IDENT & $\begin{array}{l}\text { Extrinsic Motivation - Identified, the student sees the external pathway to } \\
\text { their desires as driving their learning and performance }\end{array}$ \\
\hline AMOTIV & Amotivation, the absence of any motivation to learn \\
\hline E_PSF & $\begin{array}{l}\text { Engagement - Positive social functioning, good behavior related to feelings } \\
\text { for the activity }\end{array}$ \\
\hline E_NSF & $\begin{array}{l}\text { Engagement - Negative social functioning, bad behavior related to feelings } \\
\text { for the activity }\end{array}$ \\
\hline E_IL & $\begin{array}{l}\text { Engagement - Involvement in learning, the apprehension a student takes in } \\
\text { an activity }\end{array}$ \\
\hline E_D & $\begin{array}{l}\text { Engagement - Disposition, particular actions performed by student that } \\
\text { indicates their engagement in the activity }\end{array}$ \\
\hline $\mathrm{SE}$ & $\begin{array}{l}\text { Self-efficacy, the belief of the student that they can succeed in a particular } \\
\text { task }\end{array}$ \\
\hline
\end{tabular}

Most measures were composed of 2-4 questions that were weighted equally into an average. "Self-efficacy (SE)" was composed of 4 questions. Engagement measures including "positive social functioning (E_PSF)", "negative social functioning (E_NSF)", "dispositions (E_D)", and "involvement in learning (E_IL)" were composed of 3 questions each. Motivational measures including "intrinsic motivation - to know (IM_KNOW)", "intrinsic motivation - to accomplish (IM_ACCOMP)", “intrinsic motivation - seeking stimulation (IM_STIMUL)", "extrinsic motivation - introjected (EM_INTRO)", "extrinsic motivation - identified (EM_IDENT)", "extrinsic motivation - external regulation (EM_EXTREG)", and "amotivation (AMOTIV)" were composed of 2 questions each. Motivational and self-efficacy measures considered the field 
of wireless communications and engineering while the engagement measures considered student performance during the challenge itself.

\subsection{Results}

\section{Samples:}

Data from surveys were collected for both the 2015-2016 and 2016-2017 competitions.

Anonymous links to the survey in Qualtrics were delivered by hand to present members of all participant teams on both occasions. So long as participants had a computer or cell phone with internet access, they would be able to take part in the survey at any time after delivered the link.

For the 2015-2016 competition, there were 16 valid responses for affective measures (motivation, self-efficacy, and engagement) and 19 responses total due to lack of mandatory filling of the affective items in the survey. For the 2016-2017 competition, there were only 5 responses due to lower attendance at the finals, but the affective items were no longer optional, so all 5 responses were complete.

\section{Descriptive Statistics:}

Separate sets of means, ranges, and standard deviations are presented in the following tables for affective data. Data are presented this way to differentiate the two years to describe progression in competition outcomes through each iteration. Codes are listed in order of descending means.

Table 2. 2015-2016 Competition Affective Data

\begin{tabular}{|l|l|l|l|l|l|}
\hline Code & N & Minimum & Maximum & Mean & Std. Deviation \\
\hline SE & 16 & 4.25 & 6.00 & 5.2969 & .51006 \\
\hline E_PSF & 16 & 4.67 & 6.00 & 5.1458 & .42109 \\
\hline IM_KNOW & 16 & 3.00 & 6.00 & 5.0000 & .83666 \\
\hline EM_IDENT & 16 & 3.00 & 6.00 & 4.8438 & 1.10633 \\
\hline IM_ACCOMP & 16 & 3.50 & 6.00 & 4.6563 & .81074 \\
\hline E_IL & 16 & 1.00 & 5.67 & 4.5833 & 1.09882 \\
\hline
\end{tabular}




\begin{tabular}{|l|l|l|l|l|l|}
\hline Code & $\mathrm{N}$ & Minimum & Maximum & Mean & Std. Deviation \\
\hline IM_STIMUL & 16 & 1.50 & 5.50 & 4.2500 & 1.27802 \\
\hline E_D & 16 & 3.00 & 5.00 & 4.1042 & .71718 \\
\hline EM_INTRO & 16 & 1.00 & 6.00 & 3.2500 & 1.23828 \\
\hline EM_EXTREG & 16 & .50 & 5.50 & 3.2188 & 1.43723 \\
\hline AMOTIV & 16 & .00 & 4.50 & 1.7500 & 1.61245 \\
\hline E_NSF & 16 & .00 & 4.00 & 1.3542 & 1.15770 \\
\hline
\end{tabular}

Table 3. 2016-2017 Competition Affective Data

\begin{tabular}{|l|l|l|l|l|l|}
\hline Code & N & Minimum & Maximum & Mean & Std. Deviation \\
\hline E_PSF & 5 & 4.33 & 6.00 & 5.4667 & .69121 \\
\hline IM_KNOW & 5 & 4.50 & 6.00 & 5.4000 & .65192 \\
\hline E_IL & 5 & 4.67 & 5.33 & 5.1333 & .29814 \\
\hline E_D & 5 & 4.67 & 5.67 & 5.0667 & .43461 \\
\hline IM_ACCOMP & 5 & 2.50 & 6.00 & 4.7000 & 1.35093 \\
\hline SE & 5 & 3.75 & 5.75 & 4.7000 & .89093 \\
\hline EM_IDENT & 5 & .50 & 6.00 & 4.3000 & 2.19659 \\
\hline
\end{tabular}




\begin{tabular}{|l|l|l|l|l|l|}
\hline Code & $\mathrm{N}$ & Minimum & Maximum & Mean & Std. Deviation \\
\hline IM_STIMUL & 5 & .00 & 5.50 & 3.8000 & 2.28035 \\
\hline EM_INTRO & 5 & .00 & 5.00 & 3.1000 & 2.24722 \\
\hline EM_EXTREG & 5 & .00 & 3.50 & 1.9000 & 1.43178 \\
\hline E_NSF & 5 & .00 & 3.33 & .8667 & 1.40633 \\
\hline AMOTIV & 5 & .00 & 1.50 & .5000 & .61237 \\
\hline
\end{tabular}

\section{$\underline{\text { Free Responses }}$}

Free responses to feedback items were collected for both years of the competition. The items were structured to provide qualitative information to the competition administration team so that additional iterations of the competition could address problems and incorporate suggestions. A few excerpts that represent some of the sentiments conveyed by participants are included below.

From vocal participants (not all participants contributed feedback), sentiments generally conveyed indicated the following:

(1) Tech support by administration was effective and appreciated for prompt replies and helpfulness:

"Technical/Software support was excellent. People we were in contact during the contest were also very helpful, polite and willing to help."

(2) There were complaints of testbed nodes being down:

"Nodes were down many days[....] in some other cases we could not use our time slot."

(3) Constant changes to CRTS seemed to frustrate some. (4) Documentation may not have been clear or complete to everyone's liking:

"I would like to say that some important parts of the documentation for CRTS were really poor. Especially the beginning parts of the CRTS manual which lacks clarity [...] I am not an expert in using Ubuntu[....] The CRTS manual can be improved by providing easier and user friendly methods [to perform] various tasks." 
(5) Instructions for each phase of the competition were not always clear or timely:

"There should be some better source that we can go and see up to date instructions and Q\&A for each step. Mail group is not that efficient, since everyone cannot reach. I joined to contest in the second phase, and could not see previous instructions, [because] I was not enrolled to group at that time."

(6) Scheduling was felt to be awkward, irrational, or unfair. One explicit example of this was how 8 hour slots back-to-back would be reserved for a team on a particular node that other teams needed:

"Two 4 Hour slots a day is better than a single 8 hour slot. Towards the end, there were 28 hour slots back-to-back which proved to be very inefficient."

Another mentioned how some got scheduled through nights rather than during sensible daytime hours:

"The schedule for the competition is what bothered me the most. Most of the times, I had to work during nights and very early mornings. I hardly could work during normal working hours."

Being that it had a smaller participating group, the 2016-2017 competition survey free response data was considerably smaller. Two of the five students who participated responded to free response questions. Based on the responses that were present, the documentation provided for the participants was very helpful when beginning work on the competition that time around:

"The documentation for CRTS was very good and very helpful when I first started working on the competition."

Support services were also considered satisfactory:

“The support services were good enough for our team's purposes."

Instructions were considered well-explained, however there were concerns voiced regarding the timeliness of their delivery. One student showed some regret that the phase instructions had not been sent out sooner:

"The instructions for each phase were explained very well."

"If we would have received the phase instructions sooner, we would have had more time to change and add to our code. This would be greatly appreciated."

Testbed scheduling was not considered to be problematic outside of the familiar issue where a node will shutdown while teams are testing their code: 
"The only problem we experienced was a node going down while we were testing on the node."

"Keep as is."

In the future, students suggested "more communication about the final competition, such as the report format, and quicker feedback about phase results". They also described phases 2 and 3 of the competition as "being very similar", which was beneficial due to the timing between them. Whether to warn about changing the instructions or suggest a better time distribution for phases, the student who remarked upon this stated that their team may not have had time to complete functional code outside of these conditions.

\subsection{Discussion}

$\underline{2015-2016}$

For the 2015-2016 competition, self-efficacy was observed to be the most reportedly present measure at 5.2969, with negative social functioning (engagement) being the least reportedly present measure at 1.3542. All "positive" engagement categories (disposition, involvement in learning, positive social functioning), all intrinsic motivation categories (to know, toward accomplishment, experience stimulation), and all extrinsic motivation categories (identified, introjected, and external regulation) were reported above par (i.e. in favor of agreement with average values greater than 3 ). Amotivation was the only other measure found to be below an average of 3 on the 0 - 6 scale.

It should be noted that in terms of variations, there was less variation in general when the average found for a measure was higher, though there are some exceptions that discourage any statement of significantly-patterned relationship between mean and variance or range. Additionally, in terms of minimums and maximums, only "amotivation" and "negative social functioning" had results that were precisely 0 . A number of theoretically beneficial measures have maximums of precisely 6 .

In general, this is a largely positive indication of healthy motivation amongst the participants with regards to being in the competition. Negative social functioning would indicate that the nature of the competition bred emotionally negative conflict that can cause students to disengage from the process, demotivating participation. Amotivation would be an indicator that the students found no value in what they were doing. Extrinsic motivation through external regulation would itself be worrying if only because such a drive is rather superficial and could easily give way to amotivation if a student does not find significant reasons beyond the immediate and external to engage in learning.

\section{$\underline{2016-2017}$}

For the 2016-2017 competition, descriptive statistics show higher means of positive cognitive measures for the students involved in various respects. The highest reported measures are in positive social functioning (5.4667), intrinsic motivation to obtain knowledge (5.4), involvement 
in learning (5.1333), and disposition (5.0667). Three out of four of these measures are in engagement and one in intrinsic motivation.

Intrinsic motivation is considered preferable to extrinsic motivation in terms of finding indicators that will bring about student performance; however, identified and introjected forms of extrinsic motivation also are developmentally healthy for performance and persistence, with identified extrinsic motivation being preferable between the two. It is seen that identified extrinsic motivation in this group was higher than that of the other two extrinsic motivation categories by over 1 point at minimum.

As seen in the following table, measures with means higher than the median value on the Likert scale included most other motivational forms, self-efficacy, and engagement that are to be considered positive. The measures considered less desirable in students (amotivation, negative social functioning, and external regulation) were all measured below the median.

All participants had each engagement measure besides negative social functioning score higher than 4 overall, with the lowest score being 4.33. In self-efficacy, participants scored consistently higher than 3, with the lowest score being 3.75. The students also have a consistently great deal of intrinsic motivation to attain knowledge in the wireless communications field, as the minimum of this measure was 4.5 .

\section{$\underline{\text { Comparison }}$}

When comparing the 2016-2017 data at a glance with that of the previous year, it can be noted that self-efficacy and every category of motivational outcomes apart from two intrinsic motivation categories ("to know" and "toward accomplishment") were lower while every engagement category aside from negative social functioning rose. Alongside this, however, is the fact that multiple measures, including self-efficacy and various categories of motivation, experienced larger variances in the second year than the first year. The larger presence of intrinsic motivation as an outcome of the competition in the second year is more important to consider than drops in extrinsic motivation, since it speaks to a more reliable internal drive among the participants to persist in advanced radio engineering learning.

The sample sizes $(\mathrm{N}=16, \mathrm{~N}=5)$ could not be called representative of a general engineering student population, but if an interpretation could be set forth with this data, it would be evident that the second iteration of the competition was able to more positively involve students in the design learning process through better clarified design challenges and access to resources. The positive experiences for both groups also show that the challenge is having a positive impact on students' self-efficacy and motivation.

Observations of this improvement are further substantiated by the free response feedback in the surveys for the second year when juxtaposed with the first year. It is to be noted, however, that with so few participants in the second year of the competition, it could be that commonly reported issues like scheduling are lightened due to fewer participating teams. A smaller sample of free responses also means that there is less likely to be diversity of thought such that perspectives different from others would be present and represented in the feedback. 
There have been fewer reported issues for the 2016-2017 competition from that of 2015-2016. Based on the five participants who were present for the final competition in 2017, it is statistically evident that the students who came are well-motivated and confident in pursuing wireless communications and engineering at the end point of the challenge.

\section{Lessons Learned and Conclusions}

Technical and instructional lessons learned from the Spectrum-ShaRC competitions regarding CORNET and CRTS are outlined as follows:

Documentation

- Thorough documentation is desirable

- Some users require much more detailed documentation than others; documentation that is considered useful by advanced users may not be sufficient for all users

- High-level system descriptions as well as step-by-step instructions are needed

- The documentation was easy to follow, but still it could have been done better

Technical Limitations, Technical Support, and Maintenance

- Users expect a very high level of reliability and availability; nodes should be monitored and rebooted as needed, and bugs should be minimized

- Range of nodes may be limited if using only basic RF front end hardware (e.g., USRP and SBX daughterboards)

- User perceptions of technical support can vary

○ Consistently quick responses to questions are appreciated

- Ideally we would dedicate knowledgeable personnel to this full time so they can be very responsive.

Scheduling

- Attempts to make scheduling fair by rotating times of day for each team of users were not well received

- Administrator-assigned hours may be inconvenient for users

- Ideally allow users to self-schedule, perhaps up to a limited / arranged number of nodehours per week

We evaluate that the competition observes students being motivated to learn wireless communications. The students have shown intrinsic motivation to know about the field, according to our measures.

Consistently high self-efficacy measures for each participant show us that the contest experience makes the field of cognitive and spectrum sharing radio communications more accessible to the advanced senior and graduate students who participated.

Addressing the question of whether or not students feel integrated in the learning process of the challenge independent of their background and experience, we also see that the participants have been positively engaged while being a part of the challenge through consistently high 
engagement measures, making it evident that the contest is a beneficial experience for their engineering learning and development.

\section{Acknowledgment}

This work was made possible in part by Motorola Solutions Foundation Innovation Generation grants, by National Science Foundation award 1432416, by ONR STEM grant 11884065 . Any opinions, findings, and conclusions or recommendations expressed in this material are those of the author(s) and do not necessarily reflect the views of the sponsors.

\section{References}

[1] Executive Office of the President, President's Council of Advisors on Science and Technology, "Report to the President: Realizing the full potential of government-held spectrum to spur economic growth," Tech. Rep., 2012. [Online]. Available: https://www.whitehouse.gov/administration/eop/ostp/pcast/docsreports

[2] Defense Advanced Research Agency, DARPA Spectrum Collaboration Challenge. [Online] Available: https://spectrumcollaborationchallenge.com/. [Accessed Feb. 5, 2018].

[3] T. R. Newman, S. M. S. Hasan, D. Depoy, T. Bose, and J. H. Reed, "Designing and deploying a building-wide cognitive radio network testbed," IEEE Communications Magazine, vol. 48, iss. 9, pp. 106-112, Sept. 2010.

[4] E. Sollenberger, F. Romano, C. Dietrich, "Short Paper: Test \& Evaluation of Cognitive and Dynamic Spectrum Access Radios using The Cognitive Radio Test System," IEEE VTCFall, Boston, MA, September 6-9, 2015

[5] Mohammed, Ayat, Nicholas F. Polys, Vuk Marojevic, Richard M. Goff, and Carl B. Dietrich. "Evaluating multi-view representations of a Web3D streaming server." In Proceedings of the 22nd International Conference on 3D Web Technology, p. 5. ACM, 2017.

[6] R. J. Vallerand et al, "The Academic Motivation Scale: A Measure of Intrinsic, Extrinsic, and Amotivation in Education," Educational and Psychological Measurement, vol. 52, (4), pp. 1003-1017, 1992.

[7] P. R. Pintrich and E. V. De Groot, "Motivational and Self-Regulated Learning Components of Classroom Academic Performance," Journal of Educational Psychology, vol. 82, (1), pp. 33-40, 1990.

[8] C. Goldspink and M. Foster, "A conceptual model and set of instruments for measuring student engagement in learning," Cambridge Journal of Education, vol. 43, (3), pp. 291-311, 2013. 